\title{
Pricing under Information Asymmetry for a Large Population of Users
}

\author{
Hongxia Shen ${ }^{*}$ \\ Department of Electrical Engineering and \\ Computer Science \\ Northwestern University \\ Evanston, IL 60208, USA \\ hshen@eecs.northwestern.edu
}

\author{
Tamer Bașar \\ Department of Electrical and Computer \\ Engineering \& \\ Coordinated Science Laboratory \\ University of Illinois \\ Urbana, IL 61801, USA \\ tbasar@control.csl.uiuc.edu
}

\begin{abstract}
In this paper, we study optimal nonlinear pricing policy design for a monopolistic network service provider in the face of a large population of users. We assume that users have stochastic types. In [1], games with information symmetry have been considered; that is, users' true types may be public information available to all parties, or each user's true type may be private information known only to that user. In this paper, we study the intermediate case with information asymmetry; that is, users' true types are shared information among users, but are not disclosed to the service provider. The problem can be formulated as an incentivedesign problem, and an $\epsilon$-team optimal incentive (pricing) policy is obtained, which almost achieves Pareto optimality for the service provider. A comparative study between games with information symmetry and asymmetry are conducted as well to evaluate the service provider's game preferences.
\end{abstract}

\section{Keywords}

Nonlinear pricing, incomplete information, information asymmetry, incentives, active pricing

\section{INTRODUCTION}

In recent years, pricing has been increasingly studied for communication networks. Pricing can help to alleviate congestion. In this case, prices are essentially used as control signals from network service providers to users for them to adjust their usages of bandwidth (called flows here) accordingly. We can call this passive pricing, and the work in [2]

\footnotetext{
${ }^{*}$ Hongxia Shen was with the Coordinated Science Laboratory, University of Illinois, Urbana, IL 61801, USA, when this work was done. This work was supported in part by the National Science Foundation (NSF) under Grant ANI031976 .
}

Permission to make digital or hard copies of all or part of this work for personal or classroom use is granted without fee provided that copies are not made or distributed for profit or commercial advantage and that copies bear this notice and the full citation on the first page. To copy otherwise, to republish, to post on servers or to redistribute to lists, requires prior specific permission and/or a fee.

GameComm '07, October 22, 2007, Nantes, France

Copyright 2007 ICST 978-963-9799-00-4. stimulated much research in this direction, such as in [3], [4]. On the other hand, we can have active pricing, as considered in this paper, where prices are charged by network service providers to users in order to generate the maximal profits (which are equivalent to revenues here, since we assume that the costs are negligible or fixed). Examples of studies in this direction include [5], [6].

For active pricing, a hierarchical Stackelberg (leader-follower) game framework was proposed in [7] to study the interaction between profit-maximizing service providers and utility-maximizing users. Like most other works in the communication network pricing literature, [7] dealt with linear pricing, where what service providers announce to users are prices per unit flow which are fixed (i.e., without quantity discounts). In [8] and [1], we extended the framework, with a monopolistic network service provider, from linear pricing to general nonlinear pricing, where there may exist quantity discounts, such that the charge to a user may be a nonlinear function of the user's flow. In this context, the underlying game becomes a reverse Stackelberg game, where the service provider first needs to obtain the team solution (i.e., the action outcomes of users that jointly maximize the profit), and then solve the incentive-design problem for the optimal incentive policy (i.e., nonlinear pricing policy) such that the team solution is achieved. Precise definitions can be found in these two references. Particularly, in [8], a single user case was studied to illustrate the concept of nonlinear pricing, and in [1] the other extreme case of a large number of users was considered. We were interested in this asymptotic case because first, it allows derivation of analytical results, and second, practical communication networks generally feature a large user population.

Also, in [8] and [1], we assumed that each user may be stochastically of different true types, resulting in different utility functions, and considered the complete information game, where each user's true type is public information available to all parties, as well as the incomplete information game, where each user's true type is private information known to that user only. Moreover, for the case of multiple users, we can have the intermediate game where there is information asymmetry between the service provider and the users, so that a user's true type is shared information available to all users, but not to the service provider. This is called the partially incomplete information game in [9], in addition to the complete information game and the incomplete information game (called totally incomplete infor- 
mation game, for distinction) studied in [1]. The work [9] considered linear pricing, and here we extend the study to nonlinear pricing.

Specifically, we consider in this paper optimal nonlinear pricing policy design for a monopolistic network service provider in the face of a large number of users under partially incomplete information, and compare the results with those obtained in [1] for the other two classes of games with information symmetry. Comparison of nonlinear pricing with linear pricing for the three classes of games was reported in [10] and is not included here due to page limitation.

The rest of the paper is organized as follows. We first introduce the problem and reproduce some results of [1] in the next section. Then, in Section 3, which is the main part of this work, we study optimal nonlinear pricing policy under information asymmetry (i.e., under partially incomplete information). Following that, results are compared for the three classes of games in Section 4, and finally the paper concludes with some remarks included in Section 5 .

\section{PRICING UNDER INFORMATION SYM- METRY}

Denote the set of users by $N:=\{1, \cdots, n\}$. Following the model of [7], we formulate the net utility of User $i$ as

$$
F_{w_{i}}\left(x_{i}, \mathbf{x}_{-\mathbf{i}} ; r_{i}\right):=w_{i} \log \left(1+x_{i}\right)-\frac{1}{n-x_{i}-x_{-i}}-r_{i},
$$

for $0 \leq x_{i}<n-x_{-i}$, where $x_{i}$ is User $i$ 's flow, $\mathbf{x}_{-\mathbf{i}}:=$ $\left\{x_{j}\right\}_{j \in N, j \neq i}$ is the set of all the other users' flows, $x_{-i}:=$ $\sum_{j \in N} x_{j}-x_{i}$, and $r_{i}$ is the total charge to User $i$ by the service provider, which is allowed to depend nonlinearly on $x_{i}$. Also, let $\bar{x}:=\sum_{j \in N} x_{j}=x_{i}+x_{-i}$ and $\mathbf{x}:=\left\{x_{j}\right\}_{j \in N}$. In (1), the first term captures the user's utility for flow, which is taken to be logarithmic, with $w_{i}$ a user-specific parameter, called the type of the user. The second term is the congestion cost, which captures the delay in the framework of an $M / M / 1$ queue modeling of a link of capacity $n^{1}$. While each user tries to maximize his net utility by choosing his flow (taking the pricing policy and the other users' flows as given), the service provider needs to design optimal pricing policies such that his profit, $\bar{r}:=\sum_{j \in N} r_{j}$, is maximized.

Let $\mathbf{w}:=\left\{w_{j}\right\}_{j \in N}$ be the set of all the users' true types, and $w_{a v}:=\sum_{j \in N} w_{j} / n$. For User $i, \mathbf{w}_{-\mathbf{i}}:=\left\{w_{j}\right\}_{j \in N, j \neq i}$ is the set of all the other users' true types. In the complete information game, $\mathbf{w}$ is public information available to all parties, including the users and the service provider. In the partially incomplete information game, w is known to the users, but not to the service provider. In the totally incomplete information game, each user's true type is private information to himself; thus, User $i$ does not know $\mathbf{w}_{-\mathbf{i}}$, for $i \in N$, and the service provider does not know w. For all cases, we assume that statistical information on $\mathbf{w}$ is available and is common information to all parties. Furthermore, users are independently and identically distributed regarding their types. Suppose that for any user, there are $m$ possible types, whose set is $\left\{w^{l}\right\}_{l \in M}$, where $M:=\{1, \cdots, m\}$.

\footnotetext{
${ }^{1}$ We can see that the service provider increases the link capacity in proportion to the number of users. In [7], a more general link capacity $n c$, where $c>0$ is the per user capacity, was considered. Here we only study the special case with $c=1$, and leave the extension to more general $c$ to future work.
}

The user's type is $w^{l}$ with probability $q_{l}$ for $l \in M$, where $q_{l}>0$ and $\sum_{l \in M} q_{l}=1$. Without loss of generality, assume that $w^{1}>\cdots>w^{m}>0$.

\subsection{Complete information}

In the complete information game, $\mathbf{w}$ is known to all the players and hence no statistical information is necessary. Since the service provider knows $\mathbf{w}$, he can charge users differentially according to their true types. In order to obtain the optimal incentive policy, he needs to compute the team solution first, which is the action outcome that maximizes his profit:

$$
\left\{\left(x_{i}^{C t}(\mathbf{w}), r_{i}^{C t}(\mathbf{w})\right)\right\}_{i \in N}=\arg \max _{\left\{\left(x_{i}, r_{i}\right)\right\}_{i \in N}} \sum_{j \in N} r_{j},
$$

subject to

$$
\begin{aligned}
& F_{w_{i}}\left(x_{i}, \mathbf{x}_{-\mathbf{i}} ; r_{i}\right) \geq F_{w_{i}}\left(0, \mathbf{x}_{-\mathbf{i}} ; 0\right), \quad i \in N \\
& x_{i} \geq 0, r_{i} \geq 0, i \in N ; \quad \sum_{j \in N} x_{j}<n .
\end{aligned}
$$

Note that $r_{i}$ should be 0 for $x_{i}=0$, and (2) is the individual rationality constraint, which guarantees that the users are not worse off by participating. It was obtained in [1] that for finite but large $n$, the asymptotic team-optimal flows and charges are

$$
x_{i}^{C t}(\mathbf{w})(n) \sim \frac{2 w_{i}}{w_{a v}}-1, \quad r_{i}^{C t}(\mathbf{w})(n) \sim w_{i} \log \left(\frac{2 w_{i}}{w_{a v}}\right),
$$

for $i \in N$, if and only if

$$
w_{i}>\frac{w_{a v}}{2}, \quad \forall i \in N
$$

and the resulting team-optimal total profit is

$$
\bar{r}^{C t}(\mathbf{w})(n) \sim \sum_{j \in N} w_{j} \log \left(\frac{2 w_{j}}{w_{a v}}\right) \geq w_{a v}(\log 2) n,
$$

where the equality holds if and only if $w_{i}=w_{a v}$ for all $i \in N$.

Having obtained the team solution, the next step would be to design a pricing policy for each user, $r_{i}=\gamma_{i}\left(x_{i}\right)$, that solves the following incentive-design problem, under which individual users' utility maximizing responses lead to the team solution computed above; that is, for $i \in N$,

$$
\begin{aligned}
& x_{i}^{C t}(\mathbf{w}) \\
& =\arg \max _{x_{i}: 0 \leq x_{i}<n-x_{-i}^{C t}(\mathbf{w})} F_{w_{i}}\left(x_{i}, \mathbf{x}_{-\mathbf{i}}{ }^{C t}(\mathbf{w}) ; \gamma_{i}\left(x_{i}\right)\right),(5) \\
& \gamma_{i}\left(x_{i}^{C t}(\mathbf{w})\right)=r_{i}^{C t}(\mathbf{w}) \\
& \gamma_{i}(0)=0 .
\end{aligned}
$$

If there exists a solution to (5)-(7), which is then denoted by $\left\{\gamma_{i}^{C t}(\mathbf{w})\right\}_{i \in N}$, we say that the incentive-design problem is incentive controllable. However, it was shown in [1] that the problem is actually not incentive controllable; rather, we can find $\left\{\gamma_{i}^{C t \epsilon}(\mathbf{w})\right\}_{i \in N}$ which makes the service provider come arbitrarily close to the team-optimal profit, and so the problem is $\epsilon$-incentive controllable.

\subsection{Totally incomplete information}

With totally incomplete information, the service provider only has statistical information on $\mathbf{w}$. Thus, his objective is to maximize the expected total profit. Also, he cannot have price discrimination for different users according to their true types, which means that he should have the same 
pricing policy for all users. As a result, the team solution is the same for all the users, which consists of $m$ optimal flowcharge pairs, one pair for each possible user type, such that the expected profit is maximized. Thus, we can formulate the team problem as follows:

$$
\left\{\left(x^{l T t}, r^{l T t}\right)\right\}_{l \in M}=\arg \max _{\left\{\left(x^{l}, r^{l}\right)\right\}_{l \in M}} n \sum_{l \in M} q_{l} r^{l},
$$

subject to

$$
\begin{aligned}
& \mathcal{F}\left(w^{l}, x^{l}, r^{l} ;\left\{x^{l_{i}}\right\}\right) \geq \mathcal{F}\left(w^{l}, 0,0 ;\left\{x^{l_{i}}\right\}\right), \quad l \in M ; \\
& \mathcal{F}\left(w^{l}, x^{l}, r^{l} ;\left\{x^{l_{i}}\right\}\right) \geq \mathcal{F}\left(w^{l}, x^{k}, r^{k} ;\left\{x^{l_{i}}\right\}\right), \\
& \quad l, k \in M, l \neq k ; \\
& 0 \leq x^{l}<1, r^{l} \geq 0, \quad l \in M,
\end{aligned}
$$

where $\mathcal{F}\left(w, x, r ;\left\{y^{l_{i}}\right\}\right)$ is defined as

$$
\sum_{\left\{l_{i}\right\}_{i=1}^{n-1} \in M^{n-1}}\left\{\left(\prod_{i=1}^{n-1} q_{l_{i}}\right) F_{w}\left(x,\left\{y^{l_{i}}\right\}_{i=1}^{n-1} ; r\right)\right\} .
$$

Here, we require $x^{l}<1$ for $l \in M$, because in any case the total flow cannot exceed the total capacity $n$ for the congestion cost in (1) to be well defined. (8) is the individual rationality constraint, which guarantees that users are not worse off by participating. In addition, for incomplete information, we need (9) to induce any user with a certain type to choose the flow-charge pair desired for this type, which is called "self selection" (see [11, p. 442], [12]). Note that the individual rationality constraint is also a special kind of self-selection constraint. Also, the constraints (8) and (9) are based on the expected net utility for a user, $\mathcal{F}$, since he does not know the true types of the other $n-1$ users. In [1], a near-optimal asymptotic team solution was obtained as follows:

$$
\begin{array}{ll}
l \in M_{H}: & \tilde{x}^{l T t}=1-\delta, \quad \tilde{r}^{l T t}=w^{l_{h}} \log (2-\delta) ; \\
l \in M_{L}: & \tilde{x}^{l T t}=0, \quad \tilde{r}^{l T t}=0,
\end{array}
$$

where

$$
l_{h}=\min \left\{\arg \max _{k: k \in M} \sum_{l=1}^{k} q_{l} w^{k}\right\},
$$

$M_{H}=\left\{1, \cdots, l_{h}\right\}, M_{L}=\left\{l_{h}+1, \cdots, m\right\}$, and $\delta=a n^{-b}$ for some $a>0$ and $0<b<1$. For this near-optimal team solution, the resulting expected profit for the service provider approaches the team-optimal expected profit in the asymptotic case, which is

$$
\bar{r}^{T t}(n)=n \sum_{l \in M_{H}} q_{l} w^{l_{h}} \log 2 .
$$

Next, the incentive-design problem is to find a common incentive function for all users, $\gamma$, such that

$$
\begin{aligned}
& x^{l T t}=\arg \max _{x: x \geq 0} \mathcal{F}\left(w^{l}, x, \gamma(x) ;\left\{x^{l_{i} T t}\right\}\right), \quad l \in M \\
& \gamma\left(x^{l T t}\right)=r^{l T t}, \quad l \in M \\
& \gamma(0)=0
\end{aligned}
$$

If there exists a solution to (12)-(14), which we then denote by $\gamma^{T t}$, we say that the incentive-design problem is incentive controllable. Again, we showed in [1] that the problem is in fact $\epsilon$-incentive controllable by obtaining an $\epsilon$-team optimal incentive policy $\tilde{\gamma}^{T t \epsilon}$, which almost achieves the nearoptimal asymptotic team solution.

\section{PRICING UNDER PARTIALLY INCOM- PLETE INFORMATION}

In this section, we study optimal nonlinear pricing policy design for the partially incomplete information game. We first formulate the team problem and the incentive-design problem, and subsequently solve these two problems.

\subsection{Incentive-Design Problem Formulation}

In the partially incomplete information game, the service provider does not know $\mathbf{w}$, which is however known to the users. Thus, in order to find the team solution which maximizes the expected profit, the service provider needs to consider all the possible values of $\mathbf{w}$. In other words, suppose that User $i$ 's type is $w_{i}=w^{l_{i}}$, where $l_{i} \in M$, and then $\mathbf{l}:=\left\{l_{j}\right\}_{j \in N}$ can take any value from $M^{n}$. Therefore, the team solution consists of $n \times m^{n}$ flow-charge pairs, with one pair for each user, and totally $n$ pairs for each possible value of $\mathbf{l}$, such that the expected profit is maximized. This team problem can be formulated as follows:

$$
\begin{aligned}
& \left\{\left\{\left(x_{i}^{\mathbf{l} P t}, r_{i}^{1 P t}\right)\right\}_{i \in N}\right\}_{\mathbf{1} \in M^{n}} \\
& \quad=\arg \max _{\left\{\left\{\left(x_{i}^{\mathbf{1}}, r_{i}^{\mathbf{1}}\right)\right\}_{i \in N}\right\}_{\mathbf{1} \in M^{n}}} \sum_{\mathbf{1} \in M^{n}}\left(\prod_{j \in N} q_{l_{j}}\right) \sum_{j \in N} r_{j}^{\mathbf{1}},
\end{aligned}
$$

subject to

$$
\begin{aligned}
& F_{w^{l_{i}}}\left(x_{i}^{\mathbf{l}}, \mathbf{x}_{-\mathbf{i}}^{\mathbf{l}} ; r_{i}^{\mathbf{l}}\right) \geq F_{w^{l_{i}}}\left(0, \mathbf{x}_{-\mathbf{i}}^{\mathbf{l}} ; 0\right), \quad i \in N, \mathbf{l} \in M^{n} ; \\
& F_{w^{l_{i}}}\left(x_{i}^{\mathbf{l}}, \mathbf{x}_{-\mathbf{i}}^{\mathbf{1}} ; r_{i}^{\mathbf{l}}\right) \geq F_{w^{l_{i}}}\left(x_{j}^{\mathbf{k}}, \mathbf{x}_{-\mathbf{i}}^{\mathbf{l}} ; r_{j}^{\mathbf{k}}\right), \\
& \quad i, j \in N, \mathbf{l}, \mathbf{k} \in M^{n} ; \\
& x_{i}^{\mathbf{l}} \geq 0, r_{i}^{\mathbf{1}} \geq 0, \sum_{j \in N} x_{j}^{\mathbf{1}}<n, \quad i \in N, \mathbf{l} \in M^{n} .
\end{aligned}
$$

Note that (16) is the individual rationality constraint, which guarantees that the users are not worse off by participating, and (17) is the self-selection constraint, such that given a certain value of 1 , a user should choose the flow-charge pair desired for him in this case.

Assume that a team-optimal solution exists. Then, the incentive-design problem is to find a common incentive function, $\gamma$, for all users, since the service provider cannot differentiate users according to their types, such that for any 1 , any user's net utility is maximized at the team solution. This problem can be formulated as follows:

$$
\begin{aligned}
& x_{i}^{1 P t}=\arg \max _{x: 0 \leq x<n-x_{-i}^{1 P t}} F_{w^{l}}\left(x, x_{-i}^{1 P t} ; \gamma(x)\right), \\
& \quad i \in N, \mathbf{l} \in M^{n} ; \\
& \gamma\left(x_{i}^{1 P t}\right)=r_{i}^{1 P t}, \quad i \in N, \mathbf{l} \in M^{n} \\
& \gamma(0)=0 .
\end{aligned}
$$

If there exists a solution to (18)-(20), which is then denoted by $\gamma^{P t}$, we say that the incentive-design problem is incentive controllable.

We have thus completed the formulation of the team problem, given by (15)-(17), and the incentive-design problem, given by (18)-(20), for the partially incomplete information game. However, these two problems become intractable as $n \rightarrow \infty$, for the asymptotic case which is of particular interest to us, since the dimension of the set of possible l's, $M^{n}$, approaches infinity in this case. Therefore, in the following, we provide a simplified problem formulation for the asymptotic case. Note that as $n \rightarrow \infty$, since users are independently and identically distributed in terms of their types, 
by the Strong Law of Large Numbers [13, p. 48], with probability 1 , the fraction of those users whose types are $w^{l}$ approaches $q_{l}$ for $l \in M$. In view of this, we make the following assumption:

Assumption 1. For the asymptotic case with partially incomplete information, the service provider assumes that the number of users whose types are $w^{l}$ is $n q_{l}$, for $l \in M$, and can maximize his profit based on this assumption.

Then, by Assumption 1, the problem formulation for the team solution can be simplified such that the service provider only needs to find $m$ flow-charge pairs, one pair for each type, which maximize the total profit. Again, we take $n$ as a parameter for the asymptotic case, and the team solution can be expressed as follows ${ }^{2}$ :

$$
\begin{aligned}
& \left\{\left(x^{l P t}(n), r^{l P t}(n)\right)\right\}_{l \in M} \\
& \quad=\arg \max _{\left\{\left(x^{l}, r^{l}\right)\right\}_{l \in M}} n \sum_{l \in M} q_{l} r^{l},
\end{aligned}
$$

subject to

$$
\begin{aligned}
& F_{w^{l}}\left(x^{l}, n \sum_{h \in M} q_{h} x^{h}-x^{l} ; r^{l}\right) \geq F_{w^{l}}\left(0, n \sum_{h \in M} q_{h} x^{h}-x^{l} ; 0\right), \\
& \quad l \in M ; \\
& F_{w^{l}}\left(x^{l}, n \sum_{h \in M} q_{h} x^{h}-x^{l} ; r^{l}\right) \geq F_{w^{l}}\left(x^{k}, n \sum_{h \in M} q_{h} x^{h}-x^{l} ; r^{k}\right), \\
& \quad l, k \in M, l \neq k ; \\
& x^{l} \geq 0, r^{l} \geq 0, \quad l \in M ; \quad \sum_{l \in M} q_{l} x^{l}<1 .
\end{aligned}
$$

Assume that a solution to (21)-(23) exists. Then, the incentive-design problem is to find a common incentive function, $\gamma$, for all users, such that those users whose types are $w^{l}$ get the maximal net utilities at $\left(x^{l P t}(n), r^{l P t}(n)\right)$, for $l \in M$, which can be formulated as follows:

$$
\begin{aligned}
& x^{l P t}(n)=\arg \max _{x: 0 \leq x<n-x^{-l P t}(n)} F_{w^{l}}\left(x, x^{-l P t}(n) ; \gamma(x)\right), \\
& \quad l \in M ; \\
& \gamma\left(x^{l P t}(n)\right)=r^{l P t}(n), \quad l \in M ; \\
& \gamma(0)=0,
\end{aligned}
$$

where $x^{-l P t}(n):=n \sum_{h \in M} q_{h} x^{h P t}(n)-x^{l P t}(n)$. If there exists a solution to (24)-(26), which is then denoted by $\gamma^{P t}(n)$, we say that the incentive-design problem is incentive controllable.

Actually, we can further simplify the problem formulation under the following assumption:

Assumption 2. As $n \rightarrow \infty$, for (22), (23) and (24), assume that

$$
\lim _{n \rightarrow \infty} \frac{1}{n-n \sum_{h \in M} q_{h} x^{h}+x^{l}-x^{k}}=0, \quad l, k \in M .
$$

In other words, the congestion cost tends to zero, and thus can be neglected, for the asymptotic case with partially incomplete information.

\footnotetext{
${ }^{2}$ For the convenience of notation here, we have modified slightly the form of $F$ given in (1), such that $F_{w_{i}}\left(x_{i}, x_{-i} ; r_{i}\right):=w_{i} \log \left(1+x_{i}\right)-\left(n-x_{i}-x_{-i}\right)^{-1}-r_{i}$, for $0 \leq x_{i}<n-x_{-i}$.
}

Later, we will see that Assumption 2 is satisfied by the solution obtained. Under Assumption 2, the associated team problem, (21)-(23), can be further simplified as

$$
\left\{\left(\tilde{x}^{l P t}, \tilde{r}^{l P t}\right)\right\}_{l \in M}=\arg \max _{\left\{\left(x^{l}, r^{l}\right)\right\}_{l \in M}} n \sum_{l \in M} q_{l} r^{l},
$$

subject to

$$
\begin{aligned}
& \tilde{F}_{w^{l}}\left(x^{l} ; r^{l}\right) \geq \tilde{F}_{w^{l}}(0 ; 0), \quad l \in M ; \\
& \tilde{F}_{w^{l}}\left(x^{l} ; r^{l}\right) \geq \tilde{F}_{w^{l}}\left(x^{k} ; r^{k}\right), \quad l, k \in M, l \neq k ; \\
& x^{l} \geq 0, r^{l} \geq 0, \quad l \in M ; \quad \sum_{l \in M} q_{l} x^{l}<1,
\end{aligned}
$$

where $\tilde{F}_{w}(x ; r):=w \log (1+x)-r$. Then, the incentive-design problem, (24)-(26), can be expressed as

$$
\begin{aligned}
& \tilde{x}^{l P t}=\arg \max _{x: x \geq 0} \tilde{F}_{w^{l}}(x ; \gamma(x)), \quad l \in M ; \\
& \gamma\left(\tilde{x}^{l P t}\right)=\tilde{r}^{l P t}, \quad l \in M ; \\
& \gamma(0)=0 .
\end{aligned}
$$

If the problem is incentive controllable, then the solution is denoted by $\tilde{\gamma}^{P t}$.

\subsection{Team Solution}

\subsubsection{Optimization problem decomposition}

Next, we solve (27)-(30) for the asymptotic team solution. As in the case of the single user with incomplete information, we decompose the problem such that the optimal flows can be obtained first, followed by the optimal charges. First we have the following two lemmas, followed by two propositions, leading to the main result captured in Theorem 1.

LEMMA 1. $\tilde{x}^{1 P t} \geq \cdots \geq \tilde{x}^{m P t}$.

Proof. Fix any $l, k \in M$ such that $l<k$. By assumption, $w^{l}>w^{k}$. From (29), $\tilde{F}_{w^{l}}\left(x^{l} ; r^{l}\right) \geq \tilde{F}_{w^{l}}\left(x^{k} ; r^{k}\right)$, and $\tilde{F}_{w^{k}}\left(x^{k} ; r^{k}\right) \geq \tilde{F}_{w^{k}}\left(x^{l} ; r^{l}\right)$. By summing the two inequalities and rearranging the terms, we obtain $\left(w^{l}-w^{k}\right)\left[\log \left(1+x^{l}\right)-\right.$ $\left.\log \left(1+x^{k}\right)\right] \geq 0$, which implies $x^{l} \geq x^{k}$.

Lemma 2. Suppose that (29) holds. If (28) holds for $l=$ $m$, then it automatically holds for $l \in M /\{m\}$.

Proof. Fix any $l, k \in M$ such that $l<k$. By assumption, $w^{l}>w^{k}$, and thus $\tilde{F}_{w^{l}}\left(x^{k} ; r^{k}\right) \geq \tilde{F}_{w^{k}}\left(x^{k} ; r^{k}\right)$. Then from $(29), \tilde{F}_{w^{l}}\left(x^{l} ; r^{l}\right) \geq \tilde{F}_{w^{k}}\left(x^{k} ; r^{k}\right)$. Note that $\tilde{F}_{w^{l}}(0 ; 0)=$ $\tilde{F}_{w^{k}}(0 ; 0)=0$. Thus, if (28) holds for $k$, it also holds for $l$. Finally, we only need (28) to hold for $m$.

Proposition 1. The optimization problem for the asymptotic team solution, (27)-(30), is equivalent to the following optimization problem: $\max n \sum_{l \in M} q_{l} r^{l}$ subject to

$$
\begin{aligned}
& x^{1} \geq \cdots \geq x^{m} \geq 0 ; \\
& \sum_{l \in M} q_{l} x^{l}<1 ; \\
& r^{l} \geq 0, \quad l \in M ; \\
& r^{m} \leq w^{m} \log \left(1+x^{m}\right) ; \\
& w^{l+1} \log \frac{1+x^{l}}{1+x^{l+1}} \leq r^{l}-r^{l+1} \leq w^{l} \log \frac{1+x^{l}}{1+x^{l+1}}, \\
& \quad l \in M /\{m\} .
\end{aligned}
$$


Proof. Expressions (34)-(36) come directly from (30) and Lemma 1. By Lemma 2, (28) can be written as (37). Next, we prove that (29) can be written as (38). Fix $l$, $k$ and $h \in M$ such that $l<k<h$. By assumption, $w^{l}>w^{k}>w^{h}$. By (34), $x^{l} \geq x^{k} \geq x^{h}$. Suppose that $\tilde{F}_{w^{l}}\left(x^{l} ; r^{l}\right) \geq \tilde{F}_{w^{l}}\left(x^{k} ; r^{k}\right)$ and $\tilde{\tilde{F}}_{w^{k}}\left(x^{k} ; r^{k}\right) \geq \tilde{F}_{w^{k}}\left(x^{h} ; r^{h}\right)$. Summing the second inequality with $\left(w^{l}-w^{\bar{k}}\right) \log \left(1+x^{k}\right) \geq$ $\left(w^{l}-w^{k}\right) \log \left(1+x^{h}\right)$, which comes from $w^{l}>w^{k}$ and $x^{k} \geq x^{h}$, we get $\tilde{F}_{w^{l}}\left(x^{k} ; r^{k}\right) \geq \tilde{F}_{w^{l}}\left(x^{h} ; r^{h}\right)$. Therefore, $\tilde{F}_{w^{l}}\left(x^{l} ; r^{l}\right) \geq \tilde{F}_{w^{l}}\left(x^{h} ; r^{h}\right)$, which means that if (29) holds for the pairs $(l, k)$ and $(k, h)$, then it is also satisfied for the pair $(l, h)$. One the other hand, suppose that $\tilde{F}_{w^{h}}\left(x^{h} ; r^{h}\right) \geq$ $\tilde{F}_{w^{h}}\left(x^{k} ; r^{k}\right)$ and $\tilde{F}_{w^{k}}\left(x^{k} ; r^{k}\right) \geq \tilde{F}_{w^{k}}\left(x^{l} ; r^{l}\right)$. Adding the second inequality with

$$
-\left(w^{k}-w^{h}\right) \log \left(1+x^{k}\right) \geq-\left(w^{k}-w^{h}\right) \log \left(1+x^{l}\right),
$$

which comes from $w^{k}>w^{h}$ and $x^{k} \leq x^{l}$, we get $\tilde{F}_{w^{h}}\left(x^{k} ; r^{k}\right)$ $\geq \tilde{F}_{w^{h}}\left(x^{l} ; r^{l}\right)$. Therefore, $\tilde{F}_{w^{h}}\left(x^{h} ; r^{h}\right) \geq \tilde{F}_{w^{h}}\left(x^{l} ; r^{l}\right)$, which means that if (29) holds for the pairs $(h, k)$ and $(k, l)$, then it must also hold for the pair $(h, l)$. In conclusion, $(29)$ can be reduced to

$$
\begin{aligned}
& \tilde{F}_{w^{l}}\left(x^{l} ; r^{l}\right) \geq \tilde{F}_{w^{l}}\left(x^{l+1} ; r^{l+1}\right) ; \\
& \tilde{F}_{w^{l+1}}\left(x^{l+1} ; r^{l+1}\right) \geq \tilde{F}_{w^{l+1}}\left(x^{l} ; r^{l}\right), \quad l \in M /\{m\},
\end{aligned}
$$

which can be equivalently written as (38).

Obviously, to maximize $n \sum_{l \in M} q_{l} r^{l}, r^{m}$ should equal the upper bound in (37), and $r^{l}-r^{l-1}$ should equal the upper bound in (38), for $l \in M /\{m\}$, such that $r^{l}$ 's take the largest possible values. It can be easily seen that these values satisfy (36). Thus, we have the following proposition as a direct result of Proposition 1, whose proof is omitted here:

Proposition 2. The optimization problem for the asymptotic team solution, (27)-(30), is equivalent to $\max n \sum_{l \in M} q_{l} r^{l}$ subject to

$$
\begin{aligned}
& x^{1} \geq \cdots \geq x^{m} \geq 0 ; \\
& \sum_{l \in M} q_{l} x^{l}<1 ; \\
& r^{m}=w^{m} \log \left(1+x^{m}\right) ; \\
& r^{l}=w^{l} \log \left(1+x^{l}\right)-\sum_{k=l+1}^{m}\left(w^{k-1}-w^{k}\right) \log \left(1+x^{k}\right), \\
& \quad l \in M /\{m\} .
\end{aligned}
$$

Immediately from Proposition 2, the team solution can be decomposed as follows:

THEOREM 1. The optimization problem for the asymptotic team solution, (27)-(30), can be decomposed, such that the optimal flows can be obtained from the following problem first:

$$
\begin{aligned}
\left\{\tilde{x}^{l P t}\right\}_{l \in M}=\arg \max _{\left\{x^{l}\right\}_{l \in M}: x^{1} \geq \cdots \geq x^{m} \geq 0} n \tilde{r}_{a v}^{P} & \\
\text { s.t. } & \sum_{l \in M} q_{l} x^{l}<1
\end{aligned}
$$

where $\bar{q}_{l}:=\sum_{k=1}^{l} q_{k}$ for $l \in M, \bar{q}_{0}:=0, w^{0}:=0$, and

$$
\tilde{r}_{a v}^{P}:=\sum_{l \in M}\left(\bar{q}_{l} w^{l}-\bar{q}_{l-1} w^{l-1}\right) \log \left(1+x^{l}\right) .
$$

Then, the optimal charges, $\left\{\tilde{r}^{l P t}\right\}_{l \in M}$, can be calculated from the optimal flows, $\left\{\tilde{x}^{l P t}\right\}_{l \in M}$, by (41) and (42).

\subsubsection{Asymptotic optimal flows with relaxed constr- aint}

We first solve (43) and (44) for the asymptotic optimal flows. In order to apply the Lagrange multiplier method [14], [15], here we first relax the constraint (44) to $\sum_{l \in M} q_{l} x^{l} \leq 1$. Also, let $y^{m}=x^{m}$, and $y^{l}=x^{l}-x^{l+1}$ for $l \in M /\{m\}$, or equivalently, $x^{l}=\sum_{k=l}^{m} y^{k}$ for $l \in M$. Then, the constraint $x^{1} \geq \cdots \geq x^{m} \geq 0$ becomes $y^{l} \geq 0$ for $l \in M$, and (43) and (44) can be rewritten as

$$
\begin{array}{rl}
\max _{\left\{y^{l}: y^{l} \geq 0\right\}_{l \in M}} & n \tilde{r}_{a v}^{P}, \\
\text { s. t. } & \sum_{l \in M} \bar{q}_{l} y^{l} \leq 1,
\end{array}
$$

where

$$
\tilde{r}_{a v}^{P}=\sum_{l \in M}\left(\bar{q}_{l} w^{l}-\bar{q}_{l-1} w^{l-1}\right) \log \left(1+\sum_{k=l}^{m} y^{k}\right) .
$$

Now, for some $\lambda \geq 0$, define the Lagrangian function

$$
L\left(y^{1}, \cdots, y^{m} ; \lambda\right):=\tilde{r}_{a v}^{P}-\lambda\left(\sum_{l \in M} \bar{q}_{l} y^{l}-1\right) .
$$

Then, we obtain that the optimal solution must satisfy

$$
\begin{aligned}
& \frac{\partial}{\partial y^{l}} L\left(y^{1}, \cdots, y^{m} ; \lambda\right) \\
& =\sum_{h=1}^{l}\left[\frac{\bar{q}_{h} w^{h}-\bar{q}_{h-1} w^{h-1}}{1+\sum_{k=h}^{m} y^{k}}-\lambda q_{h}\right] \leq 0, \quad l \in M ; \\
& y^{l} \cdot \frac{\partial}{\partial y^{l}} L\left(y^{1}, \cdots, y^{m} ; \lambda\right)=0, \quad l \in M ; \\
& \lambda\left(\sum_{l \in M} \bar{q}_{l} y^{l}-1\right)=0 ; \\
& \sum_{l \in M} \bar{q}_{l} y^{l} \leq 1 ; \quad y^{l} \geq 0, l \in M ; \quad \lambda \geq 0 .
\end{aligned}
$$

It can be easily seen that $\partial L\left(y^{1}, \cdots, y^{m} ; \lambda\right) / \partial y^{1}>0$ if $\lambda=$ 0 , which contradicts (45). Thus, we must have $\lambda>0$, and (47) and (48) can be revised to

$$
\sum_{l \in M} \bar{q}_{l} y^{l}=1 ; \quad y^{l} \geq 0, l \in M ; \quad \lambda>0 .
$$

Let $\left\{l_{1}, \cdots, l_{K}\right\}$ be a subset of $M$, such that $1 \leq l_{1}<\cdots<$ $l_{K} \leq m, y^{l_{k}}>0$ for $k=1, \cdots, K$, and $y^{l}=0$ for any other $l \in M$. Define $l_{0}:=0$. Then, for $y^{l_{k}}, k=1, \cdots, K,(45)$, (46) and (49) can be reduced to

$$
\begin{aligned}
& \frac{\bar{q}_{l_{k}} w^{l_{k}}-\bar{q}_{l_{k-1}} w^{l_{k-1}}}{1+\sum_{h=k}^{K} y^{l_{h}}}-\lambda\left(\bar{q}_{l_{k}}-\bar{q}_{l_{k-1}}\right)=0, \\
& \quad k=1, \cdots, K ; \\
& \sum_{k=1}^{K} \bar{q}_{l_{k}} y^{l_{k}}=1 ; y^{l_{k}}>0, k=1, \cdots, K ; \quad \lambda>0 .
\end{aligned}
$$

By $(50)$,

$$
\sum_{h=k}^{K} y^{l_{h}}=\frac{\bar{q}_{l_{k}} w^{l_{k}}-\bar{q}_{l_{k-1}} w^{l_{k-1}}}{\lambda\left(\bar{q}_{l_{k}}-\bar{q}_{l_{k-1}}\right)}-1, \quad k=1, \cdots, K,
$$


and thus

$$
\begin{aligned}
y^{l_{k}} & =\frac{1}{\lambda}\left(\frac{\bar{q}_{l_{k}} w^{l_{k}}-\bar{q}_{l_{k-1}} w^{l_{k-1}}}{\bar{q}_{l_{k}}-\bar{q}_{l_{k-1}}}-\frac{\bar{q}_{l_{k+1}} w^{l_{k+1}}-\bar{q}_{l_{k}} w^{l_{k}}}{\bar{q}_{l_{k+1}}-\bar{q}_{l_{k}}}\right), \\
& k=1, \cdots, K-1 ; \\
y^{l_{K}} & =\frac{1}{\lambda} \cdot \frac{\bar{q}_{l_{K}} w^{l_{K}}-\bar{q}_{l_{K-1}} w^{l_{K-1}}}{\bar{q}_{l_{K}}-\bar{q}_{l_{K-1}}}-1
\end{aligned}
$$

Also, by substituting (52) into (51), we get

$1=\sum_{k=1}^{K} \bar{q}_{l_{k}} y^{l_{k}}=\sum_{k=1}^{K}\left(\sum_{h=k}^{K} y^{l_{h}}\right)\left(\bar{q}_{l_{k}}-\bar{q}_{l_{k-1}}\right)=\frac{1}{\lambda} \bar{q}_{l_{K}} w^{l_{K}}-\bar{q}_{l_{K}}$,

and thus

$$
\lambda=\frac{\bar{q}_{l_{K}} w^{l_{K}}}{1+\bar{q}_{l_{K}}} .
$$

Obviously, $\lambda>0$. From (53) and (54), for $y^{l_{k}}>0, k=$ $1, \cdots, K$, we need

$$
\begin{aligned}
& \frac{\bar{q}_{l_{k}} w^{l_{k}}-\bar{q}_{l_{k-1}} w^{l_{k-1}}}{\bar{q}_{l_{k}}-\bar{q}_{l_{k-1}}}>\frac{\bar{q}_{l_{k+1}} w^{l_{k+1}}-\bar{q}_{l_{k}} w^{l_{k}}}{\bar{q}_{l_{k+1}}-\bar{q}_{l_{k}}}, \\
& \quad k=1, \cdots, K-1 ; \\
& \frac{\bar{q}_{l_{K}} w^{l_{K}}}{1+\bar{q}_{l_{K}}}>\frac{\bar{q}_{l_{K-1}} w^{l_{K-1}}}{1+\bar{q}_{l_{K-1}}} .
\end{aligned}
$$

Now for $l \in M /\left\{l_{1}, \cdots, l_{K}\right\}, y^{l}=0$, and thus we only need (45) to hold for these $y^{l}$ 's. Given the fact that the equality holds for (45) when $l=l_{k-1}$ for $k=1, \cdots, K$, then for $l_{k-1}<l<l_{k},(45)$ can be reduced to

$$
\begin{aligned}
& \sum_{h=l_{k-1}+1}^{l}\left[\frac{\bar{q}_{h} w^{h}-\bar{q}_{h-1} w^{h-1}}{1+\sum_{j=k}^{K} y^{l_{j}}}-\lambda q_{h}\right] \\
& =\frac{\bar{q}_{l} w^{l}-\bar{q}_{l_{k-1}} w^{l_{k-1}}}{1+\sum_{j=k}^{K} y^{l_{j}}}-\lambda\left(\bar{q}_{l}-\bar{q}_{l_{k-1}}\right) \leq 0 .
\end{aligned}
$$

Combining this with (52), we get

$$
\begin{gathered}
\frac{\bar{q}_{l} w^{l}-\bar{q}_{l_{k-1}} w^{l_{k-1}}}{\bar{q}_{l}-\bar{q}_{l_{k-1}}} \leq \frac{\bar{q}_{l_{k}} w^{l_{k}}-\bar{q}_{l_{k-1}} w^{l_{k-1}}}{\bar{q}_{l_{k}}-\bar{q}_{l_{k-1}}}, \\
l_{k-1}<l<l_{k}, \quad k=1, \cdots, K .
\end{gathered}
$$

Also, since the equality holds for (45) when $l=l_{K}$, for $l_{K}<l \leq m,(45)$ can be reduced to

$$
\begin{aligned}
& \sum_{h=l_{K}+1}^{l}\left[\bar{q}_{h} w^{h}-\bar{q}_{h-1} w^{h-1}-\lambda q_{h}\right] \\
& \quad=\left(\bar{q}_{l} w^{l}-\bar{q}_{l_{K}} w^{l_{K}}\right)-\lambda\left(\bar{q}_{l}-\bar{q}_{l_{K}}\right) \leq 0 .
\end{aligned}
$$

Combining this with (55), we obtain

$$
\frac{\bar{q}_{l} w^{l}}{1+\bar{q}_{l}} \leq \frac{\bar{q}_{l_{K}} w^{l_{K}}}{1+\bar{q}_{l_{K}}}, \quad l_{K}<l \leq m
$$

Now, having obtained the optimal $y^{l}$ 's, we go back to compute $x^{l}$ s. Recall that $x^{l}=\sum_{k=l}^{m} y^{k}$ for $l \in M$. Therefore, from (52) and (55),

$$
\begin{aligned}
& x^{l}=x^{l_{k}}=\frac{1+\bar{q}_{l_{K}}}{\bar{q}_{l_{K}} w^{l_{K}}} \cdot \frac{\bar{q}_{l_{k}} w^{l_{k}}-\bar{q}_{l_{k-1}} w^{l_{k-1}}}{\bar{q}_{l_{k}}-\bar{q}_{l_{k-1}}}-1, \\
& \quad l_{k-1}<l \leq l_{k}, \quad k=1, \cdots, K \\
& x^{l}=0, \quad l_{K}<l \leq m .
\end{aligned}
$$

In conclusion, the asymptotic optimal flows with the relaxed constraint $\sum_{l \in M} q_{l} x^{l} \leq 1$ are given in (60) and (61), with (56)-(59) being the necessary and sufficient condition for optimality.

\subsubsection{An inductive method for $\left\{l_{1}, \cdots, l_{K}\right\}$}

Now, we discuss the determination of the set $\left\{l_{1}, \cdots, l_{K}\right\}$ based on (56)-(59). First, we have the following proposition:

Proposition 3. The conditions for $\left\{l_{1}, \cdots, l_{K}\right\}$, (56)(59), are equivalent to

$$
\begin{aligned}
& \frac{\bar{q}_{l} w^{l}-\bar{q}_{l_{k-1}} w^{l_{k-1}}}{\bar{q}_{l}-\bar{q}_{l_{k-1}}} \leq \frac{\bar{q}_{l_{k}} w^{l_{k}}-\bar{q}_{l_{k-1}} w^{l_{k-1}}}{\bar{q}_{l_{k}}-\bar{q}_{l_{k-1}}}, \\
& l_{k-1}<l<l_{k}, \quad k=1, \cdots, K ; \\
& \frac{\bar{q}_{l} w^{l}-\bar{q}_{l_{k-1}} w^{l_{k-1}}}{\bar{q}_{l}-\bar{q}_{l_{k-1}}}<\frac{\bar{q}_{l_{k}} w^{l_{k}}-\bar{q}_{l_{k-1}} w^{l_{k-1}}}{\bar{q}_{l_{k}}-\bar{q}_{l_{k-1}}}, \\
& l_{k}<l \leq m, k=1, \cdots, K ; \\
& \frac{\bar{q}_{l_{K-1}} w^{l_{K-1}}}{1+\bar{q}_{l_{K-1}}}<\frac{\bar{q}_{l_{K}} w^{l_{K}}}{1+\bar{q}_{l_{K}}} ; \\
& \frac{\bar{q}_{l} w^{l}}{1+\bar{q}_{l}} \leq \frac{\bar{q}_{l_{K}} w^{l_{K}}}{1+\bar{q}_{l_{K}}}, \quad l_{K}<l \leq m .
\end{aligned}
$$

Proof. First, suppose that (56)-(59) hold. We want to prove that (62)-(65) hold as well. Note that (62), (64) and (65) are just (58), (57) and (59), respectively. Thus, we only need to prove (63). For $l_{k}<l \leq m, k=1, \cdots, K$, we discuss this for different cases of $l$. If $l_{h-1}<l \leq l_{h}$ for some $h, k<h \leq K$, then from (56), we can deduce

$$
\frac{\bar{q}_{l_{j}} w^{l_{j}}-\bar{q}_{l_{j-1}} w^{l_{j-1}}}{\bar{q}_{l_{j}}-\bar{q}_{l_{j-1}}}<\frac{\bar{q}_{l_{k}} w^{l_{k}}-\bar{q}_{l_{k-1}} w^{l_{k-1}}}{\bar{q}_{l_{k}}-\bar{q}_{l_{k-1}}}, \quad j>k .
$$

Also, by (58),

$$
\begin{aligned}
\frac{\bar{q}_{l} w^{l}-\bar{q}_{l_{h-1}} w^{l_{h-1}}}{\bar{q}_{l}-\bar{q}_{l_{h-1}}} & \leq \frac{\bar{q}_{l_{h}} w^{l_{h}}-\bar{q}_{l_{h-1}} w^{l_{h-1}}}{\bar{q}_{l_{h}}-\bar{q}_{l_{h-1}}} \\
& <\frac{\bar{q}_{l_{k}} w^{l_{k}}-\bar{q}_{l_{k-1}} w^{l_{k-1}}}{\bar{q}_{l_{k}}-\bar{q}_{l_{k-1}}} .
\end{aligned}
$$

Thus,

$$
\begin{aligned}
& \frac{\bar{q}_{l} w^{l}-\bar{q}_{l_{k-1}} w^{l_{k-1}}}{\bar{q}_{l}-\bar{q}_{l_{k-1}}} \\
& =\frac{\bar{q}_{l} w^{l}-\bar{q}_{l_{h-1}} w^{l_{h-1}}+\sum_{j=k}^{h-1}\left(\bar{q}_{l_{j}} w^{l_{j}}-\bar{q}_{l_{j-1}} w^{l_{j-1}}\right)}{\bar{q}_{l}-\bar{q}_{l_{h-1}}+\sum_{j=k}^{h-1}\left(\bar{q}_{l_{j}}-\bar{q}_{l_{j-1}}\right)} \\
& <\frac{\bar{q}_{l_{k}} w^{l_{k}}-\bar{q}_{l_{k-1}} w^{l_{k-1}}}{\bar{q}_{l_{k}}-\bar{q}_{l_{k-1}}} .
\end{aligned}
$$

The other possible case is $l_{K}<l \leq m$. By (59),

$$
\frac{\bar{q}_{l} w^{l}-\bar{q}_{l_{K}} w^{l_{K}}}{\bar{q}_{l}-\bar{q}_{l_{K}}} \leq \frac{\bar{q}_{l_{K}} w^{l_{K}}}{\bar{q}_{l_{K}}} .
$$

On the other hand, by (57),

$$
\frac{\bar{q}_{l} w^{l}-\bar{q}_{l_{K}} w^{l_{K}}}{\bar{q}_{l}-\bar{q}_{l_{K}}} \leq \frac{\bar{q}_{l_{K}} w^{l_{K}}}{\bar{q}_{l_{K}}}<\frac{\bar{q}_{l_{K}} w^{l_{K}}-\bar{q}_{l_{K-1}} w^{l_{K-1}}}{\bar{q}_{l_{K}}-\bar{q}_{l_{K-1}}},
$$


which implies ${ }^{3}$

$$
\begin{aligned}
& \frac{\bar{q}_{l} w^{l}-\bar{q}_{l_{k-1}} w^{l_{k-1}}}{\bar{q}_{l}-\bar{q}_{l_{k-1}}} \\
& =\frac{\bar{q}_{l} w^{l}-\bar{q}_{l_{K}} w^{l_{K}}+\sum_{j=k}^{K}\left(\bar{q}_{l_{j}} w^{l_{j}}-\bar{q}_{l_{j-1}} w^{l_{j-1}}\right)}{\bar{q}_{l}-\bar{q}_{l_{K}}+\sum_{j=k}^{K}\left(\bar{q}_{l_{j}}-\bar{q}_{l_{j-1}}\right)} \\
& <\frac{\bar{q}_{l_{k}} w^{l_{k}}-\bar{q}_{l_{k-1}} w^{l_{k-1}}}{\bar{q}_{l_{k}}-\bar{q}_{l_{k-1}}} .
\end{aligned}
$$

Finally, we conclude that (63) holds.

For the reverse direction, suppose that (62)-(65) hold. Then, we only need to prove (56). By (63),

$$
\begin{aligned}
& \frac{\bar{q}_{l_{k}} w^{l_{k}}-\bar{q}_{l_{k-1}} w^{l_{k-1}}}{\bar{q}_{l_{k}}-\bar{q}_{l_{k-1}}} \\
& >\frac{\bar{q}_{l_{k+1}} w^{l_{k+1}}-\bar{q}_{l_{k-1}} w^{l_{k-1}}}{\bar{q}_{l_{k+1}}-\bar{q}_{l_{k-1}}} \\
& =\frac{\bar{q}_{l_{k+1}} w^{l_{k+1}}-\bar{q}_{l_{k}} w^{l_{k}}+\bar{q}_{l_{k}} w^{l_{k}}-\bar{q}_{l_{k-1}} w^{l_{k-1}}}{\bar{q}_{l_{k+1}}-\bar{q}_{l_{k}}+\bar{q}_{l_{k}}-\bar{q}_{l_{k-1}}}, \\
& \quad k=1, \cdots, K-1 .
\end{aligned}
$$

which immediately implies (56).

Based on Proposition 3, now we can determine $l_{k}$ 's inductively. Recall that $l_{0}=0, \bar{q}_{0}=0$ and $w^{0}=0$. Then, given $l_{k-1}, k=1, \cdots, K$, by (62) and (63),

$$
\begin{gathered}
l_{k}=\max \left\{\arg \max _{l: l_{k-1}<l \leq m} \frac{\bar{q}_{l} w^{l}-\bar{q}_{l_{k-1}} w^{l_{k-1}}}{\bar{q}_{l}-\bar{q}_{l_{k-1}}}\right\}, \\
k=1, \cdots, K .
\end{gathered}
$$

We have one remark here: It can be easily seen that $l_{1}=1$, since $w^{1}>\cdots>w^{m}>0$ by assumption. Next, we check whether $l_{k}$ satisfies (64) and (65) or not. If that is the case, then $k=K$ and we stop; otherwise, we proceed to determine $l_{k+1}$ by (66). Actually, we have the following proposition for $l_{K}$ :

\section{Proposition 4.}

$$
l_{K}=\min \left\{\arg \max _{l: l \in M} \frac{\bar{q}_{l} w^{l}}{1+\bar{q}_{l}}\right\} .
$$

Proof. Let $\bar{l}:=\min \left\{\arg \max _{l: l \in M} \bar{q}_{l} w^{l} /\left(1+\bar{q}_{l}\right)\right\}$. Obviously, we must have $l_{K} \geq \bar{l}$, since otherwise (65) cannot be satisfied. Next, we show $l_{K}=\bar{l}$. First, suppose that $\bar{l}=1$. Given $l_{0}=0$, we have verified that $l_{1}=1$ by (62) and (63), or equivalently, by (66). Since $\bar{l}=1$, according to the definition of $\bar{l},(64)$ and (65) also hold for $l_{1}=1$. Therefore, $K=1$ and $l_{K}=l_{1}=\bar{l}=1$. On the other hand, if $\bar{l}>1$, then given $1 \leq l_{k-1}<\bar{l}$, according to the definition of $\bar{l}$,

$$
0<\frac{\bar{q}_{l_{k-1}} w^{l_{k-1}}}{1+\bar{q}_{l_{k-1}}}<\frac{\bar{q}_{\bar{l}} w^{\bar{l}}}{1+\bar{q}_{\bar{l}}},
$$

which implies

$$
\frac{\bar{q}_{\bar{l}} w^{\bar{l}}-\bar{q}_{l_{k-1}} w^{l_{k-1}}}{\bar{q}_{\bar{l}}-\bar{q}_{l_{k-1}}}>\frac{\bar{q}_{\bar{l}} w^{\bar{l}}}{1+\bar{q}_{\bar{l}}} .
$$

${ }^{3}$ The only exception happens when $l_{K}=1$. For this case, the last two terms in the previous line are actually equal. However, since $w^{1}$ is larger than all the other $w^{l}$ 's by assumption, we can still verify the result to be proved in the following line.
Then for $l>\bar{l}$, again by the definition of $\bar{l}$,

$$
\frac{\bar{q}_{l} w^{l}}{1+\bar{q}_{l}} \leq \frac{\bar{q}_{\bar{l}} w^{\bar{l}}}{1+\bar{q}_{\bar{l}}},
$$

and thus

$$
\frac{\bar{q}_{l} w^{l}-\bar{q}_{\bar{l}} w^{\bar{l}}}{\bar{q}_{l}-\bar{q}_{\bar{l}}} \leq \frac{\bar{q}_{\bar{l}} w^{\bar{l}}}{1+\bar{q}_{\bar{l}}}<\frac{\bar{q}_{\bar{l}} w^{\bar{l}}-\bar{q}_{l_{k-1}} w^{l_{k-1}}}{\bar{q}_{\bar{l}}-\bar{q}_{l_{k-1}}} .
$$

Therefore,

$$
\begin{aligned}
\frac{\bar{q}_{l} w^{l}-\bar{q}_{l_{k-1}} w^{l_{k-1}}}{\bar{q}_{l}-\bar{q}_{l_{k-}}} & =\frac{\bar{q}_{l} w^{l}-\bar{q}_{\bar{l}} w^{\bar{l}}+\bar{q}_{\bar{l}} w^{\bar{l}}-\bar{q}_{l_{k-1}} w^{l_{k-1}}}{\bar{q}_{l}-\bar{q}_{\bar{l}}+\bar{q}_{\bar{l}}-\bar{q}_{l_{k-1}}} \\
& <\frac{\bar{q}_{\bar{l}} w^{\bar{l}}-\bar{q}_{l_{k-1}} w^{l_{k-1}}}{\bar{q}_{\bar{l}}-\bar{q}_{l_{k-1}}} .
\end{aligned}
$$

This means $l_{k} \leq \bar{l}$, since otherwise (63) cannot be satisfied. Furthermore, from the fact that $l_{K} \geq \bar{l}$, we do not stop if $l_{k}<\bar{l}$. Finally, we can always proceed to some $k$, such that $l_{k}=\bar{l}$. Then, by the definition of $\bar{l},(64)$ and (65) are satisfied for $l_{k}$, and so $k=K$ and $l_{K}=\bar{l}$.

In conclusion, the set $\left\{l_{1}, \cdots, l_{K}\right\}$ can be determined by (66) and (67) inductively.

\subsubsection{Near-optimal asymptotic team solution}

We have shown that with the relaxed constraint $\sum_{l \in M} q_{l} x^{l} \leq$ 1 , the asymptotic optimal flows can be obtained by (60) and (61). It can be seen from (49) that for these flows, $\sum_{l \in M} q_{l} x^{l}=1$. Also, the resulting maximal profit attainable with the relaxed constraint, divided by $n$, is

$$
\begin{aligned}
\tilde{r}_{a v}^{P}= & \sum_{l \in M}\left(\bar{q}_{l} w^{l}-\bar{q}_{l-1} w^{l-1}\right) \log \left(1+x^{l}\right) \\
= & \sum_{k=1}^{K} \sum_{l=l_{k-1}+1}^{l_{k}}\left(\bar{q}_{l} w^{l}-\bar{q}_{l-1} w^{l-1}\right) \log \left(1+x^{l_{k}}\right) \\
= & \sum_{k=1}^{K}\left(\bar{q}_{l_{k}} w^{l_{k}}-\bar{q}_{l_{k-1}} w^{l_{k-1}}\right) \\
& \cdot \log \left(\frac{1+\bar{q}_{l_{K}}}{\bar{q}_{l_{K}} w^{l_{K}}} \cdot \frac{\bar{q}_{l_{k}} w^{l_{k}}-\bar{q}_{l_{k-1}} w^{l_{k-1}}}{\bar{q}_{l_{k}}-\bar{q}_{l_{k-1}}}\right) \\
= & \sum_{k=1}^{K}\left(\bar{q}_{l_{k}} w^{l_{k}}-\bar{q}_{l_{k-1}} w^{l_{k-1}}\right) \log \frac{\bar{q}_{l_{k}} w^{l_{k}}-\bar{q}_{l_{k-1}} w^{l_{k-1}}}{\bar{q}_{l_{k}}-\bar{q}_{l_{k-1}}} \\
& -\bar{q}_{l_{K}} w^{l_{K}} \log \frac{\bar{q}_{l_{K}} w^{l_{K}}}{1+\bar{q}_{l_{K}}} .
\end{aligned}
$$

Now, based on (60) and (61), let

$$
\begin{aligned}
& \tilde{x}^{l P t}=\tilde{x}^{l_{k} P t}=\frac{1+\bar{q}_{l_{K}}}{\bar{q}_{l_{K}} w^{l_{K}}} \cdot \frac{\bar{q}_{l_{k}} w^{l_{k}}-\bar{q}_{l_{k-1}} w^{l_{k-1}}}{\bar{q}_{l_{k}}-\bar{q}_{l_{k-1}}}-1-\delta, \\
& \quad l_{k-1}<l \leq l_{k}, \quad k=1, \cdots, K ; \\
& \tilde{x}^{l P t}=0, \quad l_{K}<l \leq m,
\end{aligned}
$$

where $\delta=a n^{-b}$ for some $a>0$ and $0<b<1$. Next, we show that $\tilde{x}^{l P t}$ 's defined by (68) and (69) solve (43) and (44) for the asymptotic case, and thus provide the near-optimal flows for the asymptotic team solution. First, $\sum_{l \in M} q_{l} \tilde{x}^{l P t}=$ $1-\bar{q}_{l_{K}} \delta<1$, which satisfies the original constraint (44). 
Also, Assumption 2 holds, since for $l, k \in M$,

$$
\begin{aligned}
& \lim _{n \rightarrow \infty} \frac{1}{n-n \sum_{h \in M} q_{h} \tilde{x}^{h P t}+\tilde{x}^{l P t}-\tilde{x}^{k P t}} \\
& =\lim _{n \rightarrow \infty} \frac{1}{\bar{q}_{l_{K}} a n^{1-b}+\tilde{x}^{l P t}-\tilde{x}^{k P t}}=0 .
\end{aligned}
$$

Furthermore,

$$
\begin{aligned}
\tilde{r}_{a v}^{P t}:= & \sum_{l \in M}\left(\bar{q}_{l} w^{l}-\bar{q}_{l-1} w^{l-1}\right) \log \left(1+\tilde{x}^{l P t}\right) \\
= & \sum_{k=1}^{K}\left(\bar{q}_{l_{k}} w^{l_{k}}-\bar{q}_{l_{k-1}} w^{l_{k-1}}\right) \\
& \cdot\left[\log \left(1+\tilde{x}^{l_{k} P t}+\delta\right)-\left(1+\tilde{x}^{l_{k} P t}+\delta\right)^{-1} a n^{-b}\right] \\
\sim & \sum_{k=1}^{K}\left(\bar{q}_{l_{k}} w^{l_{k}}-\bar{q}_{l_{k-1}} w^{l_{k-1}}\right) \log \frac{\bar{q}_{l_{k}} w^{l_{k}}-\bar{q}_{l_{k-1}} w^{l_{k-1}}}{\bar{q}_{l_{k}}-\bar{q}_{l_{k-1}}} \\
& \quad-\bar{q}_{l_{K}} w^{l_{K}} \log \frac{\bar{q}_{l_{K}} w^{l_{K}}}{1+\bar{q}_{l_{K}}} .
\end{aligned}
$$

Therefore, for the asymptotic case, $\tilde{x}^{l P t}$ 's almost achieve the maximal profit attainable with the relaxed constraint, defined by

$$
\begin{aligned}
\bar{r}^{P t}(n) & \\
:= & n \sum_{k=1}^{K}\left(\bar{q}_{l_{k}} w^{l_{k}}-\bar{q}_{l_{k-1}} w^{l_{k-1}}\right) \log \frac{\bar{q}_{l_{k}} w^{l_{k}}-\bar{q}_{l_{k-1}} w^{l_{k-1}}}{\bar{q}_{l_{k}}-\bar{q}_{l_{k-1}}} \\
& -n \bar{q}_{l_{K}} w^{l_{K}} \log \frac{\bar{q}_{l_{K}} w^{l_{K}}}{1+\bar{q}_{l_{K}}},
\end{aligned}
$$

and so (68) and (69) provide the near-optimal flows for the asymptotic team solution. Then, the near-optimal charges for the asymptotic team solution, $\left\{\tilde{r}^{l P t}\right\}_{l \in M}$, can be obtained from $\left\{\tilde{x}^{l P t}\right\}_{l \in M}$ by (41) and (42), and finally we have

$$
\begin{aligned}
& \tilde{r}^{l P t}=\tilde{r}^{l_{k} P t} \\
& =w^{l_{k}} \log \left(1+\tilde{x}^{l_{k} P t}\right)-\sum_{h=k+1}^{K}\left(w^{l_{h-1}}-w^{l_{h}}\right) \log \left(1+\tilde{x}^{l_{h} P t}\right), \\
& \quad l_{k-1}<l \leq l_{k}, k=1, \cdots, K ; \\
& \tilde{r}^{l P t}=0, \quad l_{K}<l \leq m .
\end{aligned}
$$

\subsection{Solution of the Incentive-Design Problem}

Having obtained the near-optimal asymptotic team solution $\left\{\left(\tilde{x}^{l P t}, \tilde{r}^{l P t}\right)\right\}_{l \in M}$, we now address the incentive-design problem (31)-(33) for $\tilde{\gamma}^{P t}$. Note that $\left\{\left(\tilde{x}^{l P t}, \tilde{r}^{l P t}\right)\right\}_{l \in M}$ satisfies (41) and (42), or equivalently,

$$
\begin{aligned}
& w^{m} \log \left(1+\tilde{x}^{m P t}\right)-\tilde{r}^{m P t}=0 \\
& w^{l} \log \left(1+\tilde{x}^{l P t}\right)-\tilde{r}^{l P t}=w^{l} \log \left(1+\tilde{x}^{(l+1) P t}\right)-\tilde{r}^{(l+1) P t}, \\
& \quad l \in M /\{m\} .
\end{aligned}
$$

In particular, this implies

$$
\begin{aligned}
& \tilde{F}_{w^{l_{K}}}\left(\tilde{x}^{l_{K} P t} ; \tilde{r}^{l_{K} P t}\right)=\tilde{F}_{w^{l_{K}}}(0 ; 0)=0 \\
& \tilde{F}_{w^{l_{k}}}\left(\tilde{x}^{l_{k} P t} ; \tilde{r}^{l_{k} P t}\right)=\tilde{F}_{w^{l_{k}}}\left(\tilde{x}^{l_{k+1} P t} ; \tilde{r}^{l_{k+1} P t}\right), \\
& \quad k=1, \cdots, K-1,
\end{aligned}
$$

which means that a user with the type $w^{l_{K}}$ may choose the flow-charge pair $(0,0)$ instead of $\left(\tilde{x}^{l_{K} P t}, \tilde{r}^{l_{K} P t}\right)$, and a user with the type $w^{l_{k}}$ may choose $\left(\tilde{x}^{l_{k+1} P t}, \tilde{r}^{l_{k+1} P t}\right)$ instead of $\left(\tilde{x}^{l_{k} P t}, \tilde{r}^{l_{k} P t}\right)$, for $k=1, \cdots, K-1$. Thus, strictly speaking, the incentive-design problem (31)-(33) is not incentive controllable.

Next, we show that the problem is $\epsilon$-incentive controllable by obtaining an $\epsilon$-team optimal incentive policy, $\tilde{\gamma}^{P t \epsilon}$. First, let $\tilde{\gamma}^{P t \epsilon}(0)=0$, and $\tilde{\gamma}^{P t \epsilon}\left(\tilde{x}^{l_{K} P t}\right)=\tilde{r}^{l_{K} P t}-\epsilon_{K}$, where $\epsilon_{K}$ is some small positive number. Then,

$$
\tilde{F}_{w^{l} K}\left(\tilde{x}^{l_{K} P t} ; \tilde{\gamma}^{P t \epsilon}\left(\tilde{x}^{l_{K} P t}\right)\right)=\epsilon_{K}>0
$$

which guarantees that a user with the type $w^{l_{K}}$ will not choose $(0,0)$. On the other hand, for a user with the type $w^{l}, l_{K}<l \leq m$, to still stick to $(0,0)$, we need

$$
\begin{aligned}
& \tilde{F}_{w^{l}}\left(\tilde{x}^{l_{K} P t} ; \tilde{\gamma}^{P t \epsilon}\left(\tilde{x}^{l_{K} P t}\right)\right) \\
& =\epsilon_{K}-\left(w^{l_{K}}-w^{l}\right) \log \left(1+\tilde{x}^{l_{K} P t}\right)<0 .
\end{aligned}
$$

Next, for $k=K-1, \cdots, 1$, let $\tilde{\gamma}^{P t \epsilon}\left(\tilde{x}^{l_{k} P t}\right)=\tilde{r}^{l_{k} P t}-\epsilon_{k}$, where $\epsilon_{k}>\epsilon_{k+1}$ such that

$$
\begin{aligned}
& \tilde{F}_{w^{l_{k}}}\left(\tilde{x}^{l_{k} P t} ; \tilde{\gamma}^{P t \epsilon}\left(\tilde{x}^{l_{k} P t}\right)\right) \\
& =\tilde{F}_{w^{l_{k}}}\left(\tilde{x}^{l_{k+1} P t} ; \tilde{\gamma}^{P t \epsilon}\left(\tilde{x}^{l_{k+1} P t}\right)\right)+\epsilon_{k}-\epsilon_{k+1} \\
& >\tilde{F}_{w^{l_{k}}}\left(\tilde{x}^{l_{k+1} P t} ; \tilde{\gamma}^{P t \epsilon}\left(\tilde{x}^{l_{k+1} P t}\right)\right) .
\end{aligned}
$$

On the other hand, for a user with the type $w^{l}, l_{k}<l \leq m$, to stick to their desired choices, similarly, we need

$$
\begin{aligned}
& \left(w^{l_{k}}-w^{l_{k}+1}\right)\left[\log \left(1+\tilde{x}^{l_{k} P t}\right)-\log \left(1+\tilde{x}^{l_{k+1} P t}\right)\right], \\
& >\epsilon_{k}-\epsilon_{k+1}, \quad k=1, \cdots, K-1 .
\end{aligned}
$$

Finally, for (31) to be satisfied, that is, for $\tilde{x}^{l P t}$ to be the unique maximizing point of $\tilde{F}_{w^{l}}\left(x ; \tilde{\gamma}^{P t \epsilon}(x)\right)$ for $x \geq 0$, we need

$$
\begin{aligned}
& \tilde{\gamma}^{P t \epsilon}(x)>w^{l} \log (1+x)-\left[w^{l} \log \left(1+\tilde{x}^{l P t}\right)-\tilde{r}^{l P t}\right]-\epsilon_{l}, \\
& \quad l \in M,
\end{aligned}
$$

for $x \geq 0$ and $x \notin\left\{0, \tilde{x}^{l_{1} P t}, \cdots, \tilde{x}^{l_{K} P t}\right\}$ (for instance, we can let $\left.\tilde{\gamma}^{\overline{P t} \epsilon}(x)=w^{1} \log (1+x)\right)$. In conclusion, a user with the type $w^{l}, l \in M$, chooses the pair $\left(\tilde{x}^{l P t}, \tilde{\gamma}^{P t \epsilon}\left(\tilde{x}^{l P t}\right)\right)$, if for the incentive policy $\tilde{\gamma}^{P t \epsilon}, \tilde{\gamma}^{P t \epsilon}(0)=0, \tilde{\gamma}^{P t \epsilon}\left(\tilde{x}^{l_{k} P t}\right)=\tilde{r}^{l_{k} P t}-\epsilon_{k}$ for $k=1, \cdots, K$, where $\epsilon_{1}>\cdots>\epsilon_{K}>0$, and (73) to (75) are satisfied. Then, the resulting profit is

$$
\bar{r}^{P t}(n)-n \sum_{l \in M} q_{l} \epsilon_{l}=\bar{r}^{P t}(n)-n \sum_{k=1}^{K}\left(\bar{q}_{l_{k}}-\bar{q}_{l_{k-1}}\right) \epsilon_{k},
$$

which can come arbitrarily close to the team-optimal profit $\bar{r}^{P t}(n)$ by making $\epsilon_{k}$ 's arbitrarily small. Therefore, $\tilde{\gamma}^{P t \epsilon}$ is an $\epsilon$-team optimal incentive policy.

\section{COMPARISON OF THE THREE GAMES}

In the following, we compare results for the partially incomplete information game, given by (66), (67), and (70), with those for the other two games, given by (3) and (4) for complete information, and (10) and (11) for totally incomplete information. Note that for the asymptotic case, it is stipulated by Assumption 1 that with probability 1, the number of users whose types are $w^{l}$ is $n q_{l}$, for $l \in M$. Then for the complete information game, by (3) and (4), the asymptotic optimal profit for this case is

$$
\bar{r}^{C t}(n)=n \sum_{l=1}^{l_{C t}} q_{l} w^{l} \log w^{l}-n\left(\sum_{l=1}^{l_{C t}} q_{l} w^{l}\right) \log \frac{\sum_{l=1}^{l_{C t}} q_{l} w^{l}}{2 \bar{q}^{l} C t},
$$


where $l_{C t}$ for the smallest admissible type satisfies

$$
w^{l_{C t}}>\frac{\sum_{l=1}^{l_{C t}} q_{l} w^{l}}{2 \bar{q}^{l} C t} .
$$

We first compare the partially incomplete information game with the totally incomplete one. From the definition of $l_{h}$, for $l>l_{h}$, we must have $\bar{q}_{l} w^{l} \leq \bar{q}_{l_{h}} w^{l_{h}}$, which implies

$$
\frac{\bar{q}_{l} w^{l}}{1+\bar{q}_{l}}<\frac{\bar{q}_{l_{h}} w^{l_{h}}}{1+\bar{q}_{l_{h}}}, \quad l>l_{h} .
$$

Thus, $l_{h} \geq l_{K}$, which means that more users can be possibly admitted for the totally incomplete information game. Next, we compare $\bar{r}^{P t}(n)$ with $\bar{r}^{T t}(n)$, and discuss several possible cases. First, if $l_{K}=l_{h}=1$, then

$$
\bar{r}^{P t}(n)=n q_{1} w^{1} \log \frac{1+q_{1}}{q_{1}} \geq n q_{1} w^{l} \log 2=\bar{r}^{T t}(n) .
$$

If $l_{K}=l_{h}>1$, then we have the following proposition for $\bar{r}^{P t}(n)$, the proof of which is provided in the appendix:

Proposition 5. If $l_{K}>1$, then

$$
\bar{r}^{P t}(n)>n \bar{q}_{l_{K}} w^{l_{K}} \log \frac{1+\bar{q}_{l_{K}}}{\bar{q}_{l_{K}}} .
$$

Thus,

$$
\bar{r}^{P t}(n)>n \bar{q}_{l_{K}} w^{l_{K}} \log 2=n \bar{q}_{l_{h}} w^{l_{h}} \log 2=\bar{r}^{T t}(n) .
$$

If $l_{K}<l_{h}$, then from the definitions of $l_{K}$ and $l_{h}$, we must have

$$
\bar{q}_{l_{K}} w^{l_{K}}<\bar{q}_{l_{h}} w^{l_{h}} \leq\left(1+\bar{q}_{l_{h}}\right) \frac{\bar{q}_{l_{K}} w^{l_{K}}}{1+\bar{q}_{l_{K}}}
$$

and so

$$
\begin{aligned}
n \bar{q}_{l_{K}} w^{l_{K}} \log 2 & <\bar{r}^{T t}(n)=n \bar{q}_{l_{h}} w^{l_{h}} \log 2 \\
& \leq n\left(1+\bar{q}_{l_{h}}\right) \frac{\bar{q}_{l_{K}} w^{l_{K}}}{1+\bar{q}_{l_{K}}} \log 2
\end{aligned}
$$

which, combined with (76), implies

$$
\frac{\bar{r}^{T t}(n)}{\bar{r}^{P t}(n)} \leq\left(1+\bar{q}_{l_{h}}\right) \log 2\left[\left(1+\bar{q}_{l_{K}}\right) \log \frac{1+\bar{q}_{l_{K}}}{\bar{q}_{l_{K}}}\right]^{-1} .
$$

We can easily verify that $\left(1+\bar{q}_{l_{K}}\right) \log \left[\left(1+\bar{q}_{l_{K}}\right) / \bar{q}_{l_{K}}\right]$ is strictly convex and strictly decreasing for $0<\bar{q}_{l_{K}}<1$ (since $l_{K}<$ $l_{h}$ ), and so it is larger than the infimum, $2 \log 2$. Thus,

$$
\frac{\bar{r}^{T t}(n)}{\bar{r}^{P t}(n)}<\frac{1+\bar{q}_{l_{h}}}{2} \leq 1 .
$$

In conclusion, even though more users can possibly be admitted for the totally incomplete information game, we always have $\bar{r}^{P t}(n)>\bar{r}^{T t}(n)$, except that the equality may hold when $l_{K}=l_{h}=1$.

Next, for comparison with the complete information game, we first know that all being team-optimal with correspondingly inferior information, we have $\bar{r}^{C t}(n) \geq \bar{r}^{P t}(n) \geq \bar{r}^{T t}(n)$ However, the relative differences are difficult to express in any useful analytic form, and hence we resort to numerical analysis to evaluate the profit loss due to the two different types of incomplete information. From Table 1, we can see that for the scenarios picked (rather arbitrarily), compared with the complete information game, the profit loss due to partially incomplete information is always less (and rather substantially) than the profit loss due to totally incomplete information, which confirms our previous conclusion. The table also shows the extent of the drop in profit due to loss of information. The bottom line is that the service provider makes the most profit with complete information, while if he cannot obtain information on the users' true types, he prefers partially incomplete information for a higher profit, and it is beneficial for him to encourage users to share this information among themselves when possible. In other words, as leader in the game, the service provider is better off with better informed users (followers).

\section{CONCLUSION}

In this paper, we have obtained an $\epsilon$-team optimal incentive policy, which almost achieves Pareto optimality for a monopolistic network service provider facing a large population of users, for the partially incomplete information game. Comparison with results obtained in [1] for the complete and totally incomplete information games indicates that if the service provider is not able to obtain information on users' true types, then it is beneficial for him to encourage information sharing among users on each other's true types when possible.

Note that the above conclusion is made for general nonlinear pricing policies. One remark here is that if we restrict pricing policies to be linear, the same relationship holds; that is, the service provider makes the highest profit under complete information, followed by the profit under partially, and finally the profit under totally incomplete information. However, by turning from linear pricing policies to nonlinear ones, the service provider achieves a higher profit for all three games. A complete study of linear and nonlinear pricing under the three information scenarios can be found in [10].

\section{REFERENCES}

[1] H.-X. Shen and T. Başar, "Optimal nonlinear pricing for a monopolistic network service provider with complete and incomplete information," IEEE Journal on Selected Areas in Communications, vol. 25, no. 6, pp. 1216-1223, August 2007.

[2] F. Kelly, A. Maulloo, and D. Tan, "Rate control for communication networks: Shadow prices, proportional fairness and stability," Journal of the Operational Research Society, vol. 49, pp. 237-252, 1998.

[3] R. Johari and J. Tsitsiklis, "Efficiency loss in a network resource allocation game," Mathematics of Operations Research, vol. 29, no. 3, pp. 407-435, 2004.

[4] R. Maheswaran and T. Başar, "Efficient signal proportional allocation (ESPA) mechanisms: Decentralized social welfare maximization for divisible resources," IEEE Journal on Selected Areas in Communications, vol. 24, no. 5, pp. 1000-1009, May 2006.

[5] M. Honig and K. Steiglitz, "Usage-based pricing of packet data generated by a heterogeneous user population," in Proceedings IEEE INFOCOM 1995, 1995, vol. 2, pp. 867-874.

[6] L. He and J. Walrand, "Pricing and revenue sharing strategies for Internet service providers," IEEE Journal on Selected Areas in Communications, vol. 24, no. 5, pp. 942-951, May 2006. 
Table 1: Numerical results for comparison of asymptotic optimal profits for the three classes of games

\begin{tabular}{cc|cc|cc|cc|c|c}
\hline$q_{l}{ }^{\prime} \mathrm{s}$ & $\frac{w^{l}}{w_{a v}} ' s$ & $l_{C t}$ & $\frac{\bar{r}^{C t}(n)}{n w_{a v}}$ & $l_{K}$ & $\frac{\bar{r}^{P t}(n)}{n w_{a v}}$ & $l_{h}$ & $\frac{\bar{r}^{T t}(n)}{n w_{a v}}$ & $1-\frac{\bar{r}^{P t}(n)}{\bar{r} C t}(n)$ & $1-\frac{\bar{r}^{T t}(n)}{\bar{r} C t}(n)$ \\
\hline \hline 1 & 1 & 1 & $\log 2$ & 1 & $\log 2$ & 1 & $\log 2$ & 0 & 0 \\
\hline$\frac{1}{3}$ & $\frac{5}{4}, 1, \frac{3}{4}$ & 3 & 0.7142 & 2 & 0.6319 & 3 & 0.5199 & $11.52 \%$ & $27.21 \%$ \\
\hline$\frac{1}{2}$ & $\frac{5}{4}, \frac{3}{4}$ & 2 & 0.7247 & 1 & 0.6866 & 2 & 0.5199 & $5.26 \%$ & $28.26 \%$ \\
\hline$\frac{1}{3}$ & $\frac{4}{3}, 1, \frac{2}{3}$ & 3 & 0.7309 & 2 & 0.6486 & 2 & 0.4621 & $11.26 \%$ & $36.78 \%$ \\
\hline$\frac{1}{2}$ & $\frac{4}{3}, \frac{2}{3}$ & 2 & 0.7498 & 1 & 0.7324 & 1 & 0.4621 & $2.32 \%$ & $38.37 \%$ \\
\hline$\frac{1}{3}$ & $\uparrow \frac{3}{2}, 1, \downarrow \frac{1}{2}$ & 3 & $\uparrow 0.7804$ & 2 & $\uparrow 0.6981$ & 2 & 0.4621 & $10.55 \%$ & $40.79 \%$ \\
\hline$\frac{1}{2}$ & $\uparrow \frac{3}{2}, \downarrow \frac{1}{2}$ & 2 & $\uparrow 0.8240$ & 1 & $\uparrow 0.8240$ & 1 & $\uparrow 0.5199$ & 0 & $36.91 \%$ \\
\hline
\end{tabular}

[7] T. Başar and R. Srikant, "Revenue-maximizing pricing and capacity expansion in a many-users regime," in Proceedings IEEE INFOCOM 2002, 2002, pp. 1556-1563.

[8] H.-X. Shen and T. Başar, "Incentive-based pricing for network games with complete and incomplete information," in Annals of the International Society of Dynamic Games, Vol. 9, Advances in Dynamic Game Theory: Numerical Methods, Algorithms, and Applications to Ecology and Economics, S. Jørgensen, M. Quincampoix, and T. L. Vincent, Eds. Boston, MA: Birkhäuser, 2007, pp. 431-458.

[9] H.-X. Shen and T. Başar, "Hierarchical network games with various types of public and private information," in Proceedings of the 45th IEEE Conference on Decision and Control, 2006, pp. 2825-2830.

[10] H.-X. Shen, "Linear and nonlinear pricing for network games with complete and incomplete information," Ph.D. dissertation, University of Illinois at Urbana-Champaign, Urbana, IL, 2007.

[11] H. R. Varian, Intermediate Microeconomics: A Modern Approach, 6th Edition. New York, NY: W.W. Norton \& Company, 2003.

[12] E. Maskin and J. Riley, "Monopoly with incomplete information," The RAND Journal of Economics, vol. 15, no. 2, pp. 171-196, Summer 1984.

[13] S. R. S. Varadhan, Probability Theory. Providence, Rhode Island: American Mathematical Society, 2001.

[14] D. G. Luenberger, Optimization by Vector Space Methods. New York, NY: John Wiley \& Sons, 1969.

[15] D. P. Bertsekas, Nonlinear Programming, 2nd Edition. Belmont, MA: Athena Scientific, 1999.

\section{APPENDIX}

Given $\bar{q}_{l_{k}}$ 's and $q_{l_{K}} w^{l_{K}}$ as fixed, such that $0<\bar{q}_{l_{1}}<\cdots<$ $\bar{q}_{l_{K}} \leq 1$ and $w^{l_{K}}>0$, we study how to choose $w^{l_{k}}, k=$ $1, \cdots, K-1$, such that $\bar{r}^{P t}(n)$ is minimized. Let

$$
z_{k}:=\frac{\bar{q}_{l_{k}} w^{l_{k}}-\bar{q}_{l_{k-1}} w^{l_{k-1}}}{\bar{q}_{l_{k}}-\bar{q}_{l_{k-1}}}, \quad k=1, \cdots, K
$$

and $a_{k}:=\bar{q}_{l_{k}}-\bar{q}_{l_{k-1}}, k=1, \cdots, K$. Then by assumption, $a_{k}$ 's are fixed, and $S:=q_{l_{K}} w^{l_{K}}=\sum_{k=1}^{K} a_{k} z_{k}$ is specified as well. Thus, the above problem is equivalent to minimizing

$$
\begin{aligned}
& Z\left(z_{1}, \cdots, z_{K-1}\right) \\
& :=\sum_{k=1}^{K}\left(\bar{q}_{l_{k}} w^{l_{k}}-\bar{q}_{l_{k-1}} w^{l_{k-1}}\right) \log \frac{\bar{q}_{l_{k}} w^{l_{k}}-\bar{q}_{l_{k-1}} w^{l_{k-1}}}{\bar{q}_{l_{k}}-\bar{q}_{l_{k-1}}} \\
& =\sum_{k=1}^{K} a_{k} z_{k} \log z_{k} \\
& =\sum_{k=1}^{K-1} a_{k} z_{k} \log z_{k}+\left(S-\sum_{k=1}^{K-1} a_{k} z_{k}\right) \log \frac{S-\sum_{k=1}^{K-1} a_{k} z_{k}}{a_{K}}
\end{aligned}
$$

by choosing $z_{k}, k=1, \cdots, K-1$, such that $z_{k}>0$ for $k=1, \cdots, K$. Now,

$$
\frac{\partial}{\partial z_{k}} Z\left(z_{1}, \cdots, z_{K-1}\right)=a_{k} \log z_{k}-a_{k} \log \frac{S-\sum_{h=1}^{K-1} a_{h} z_{h}}{a_{K}}
$$

for $k=1, \cdots, K-1$, and

$$
\begin{aligned}
& \frac{\partial^{2}}{\partial z_{k}^{2}} Z\left(z_{1}, \cdots, z_{K-1}\right)=\frac{a_{k}}{z_{k}}+\frac{a_{k}^{2}}{S-\sum_{h=1}^{k-1} a_{h} z_{h}} \\
& \frac{\partial^{2}}{\partial z_{k} \partial z_{l}} Z\left(z_{1}, \cdots, z_{K-1}\right)=\frac{a_{k} a_{l}}{S-\sum_{h=1}^{k-1} a_{h} z_{h}}, \quad k \neq l .
\end{aligned}
$$

It can be easily verified that the Hessian matrix of $Z$ is positive definite, and so $Z$ is strictly convex. Thus, the unique minimizing solution can be obtained by solving the first order condition, which implies $z_{k}=\left(S-\sum_{h=1}^{K-1} a_{h} z_{h}\right) / a_{K}=$ $z_{K}$ for $k=1, \cdots, K-1$. Therefore, to minimize $\bar{r}^{P t}(n)$, $z_{k}$ 's should all be the same. As a result, $w^{l_{k}}=w^{l_{K}}$ for $k=1, \cdots, K-1$, and the infimum of $\bar{r}^{P t}(n)$ is as given on the right side of (76). However, by assumption, we must have $w^{l_{1}}>\cdots>w^{l_{K}}$. That means we cannot obtain this infimum, but can come arbitrarily close to it. Note that this can be achieved without violating (66) and (67). Finally, we conclude that (76) holds. 